\title{
Quale acqua (sotterranea) bolle in pentola
}

Marco Petitta - Presidente del Comitato Italiano IAH - marco.petitta@uniroma1.it

La fine dell'estate, per chi si occupa di acque sotterranee, ha molti motivi in più di attenzione, rispetto al classico ritorno al lavoro dopo le vacanze. Sono i mesi dei convegni e delle fiere, ma anche quelli in cui la disponibilità idrica comincia a diminuire, in attesa della ricarica invernale.

Se ci aggiungiamo i classici buoni propositi post-vacanze (quell'articolo che non si è mai scritto da mettere in cantiere per i ricercatori, l'acquisto di quello strumento per il professionista, ecc.), l'agenda si fa fitta fino all'over-booking.

La IAH italiana in settembre e ottobre è presente in molte occasioni di incontro, nazionali e internazionali. Lappuntamento classico per i soci IAH è a metà settembre a Marrakech (Marocco), dove si svolge per la prima volta in nord-Africa il Congresso Mondiale. Il congresso ha una forte connotazione connessa ai temi della salvaguardia della risorsa, degli effetti dei cambiamenti climatici e della siccità, ma come sempre in queste occasioni tutti i settori dell'idrogeologia saranno coinvolti. La "colonia" italiana è particolarmente ricca quest'anno, oltre 20 partecipanti, anche in virtù della presentazione del prossimo convegno mondiale IAH che, come ben sanno i nostri lettori, sarà ospitato a Roma dal 13 al 18 settembre 2015. I preparativi fervono e le prime indicazioni sul programma scientifico e sociale e sui principali appuntamenti previsti sono già disponibili sul sito ufficiale www.iah2015.org. Si tratta di una importantissima occasione per gli Idrogeologi italiani di "mostrarsi" alla comunità internazionale, non solo a livello di ricerca ma anche in ambito professionale, presentando le proprie esperienze e apprendendo le ultime novità tecnologiche $\mathrm{e}$ metodologiche, in modo da mettere in pratica il travaso delle conoscenze sempre necessario e auspicabile tra ricerca e professione. A tal proposito, si stanno mettendo in cantiere alcuni workshop paralleli alle classiche attività congressuali, dedicati a temi più strettamente professionali di ampio respiro, oltre a corsi brevi di formazione tenuti da esperti internazionali nei giorni immediatamente precedenti il congresso. Per essere continuamente aggiornati, approfittate dell'opzione di preregistrazione sul sito, e riceverete la newsletter periodica relativa al congresso.

In questo ambito, il nostro Comitato ha organizzato nello scorso mese di maggio il primo corso di aggiornamento sulle captazioni di pozzi e sorgenti, in collaborazione con l'Ordine dei Geologi della Lombardia. Visto il successo dell'iniziativa, e la reciproca soddisfazione dei partecipanti e degli organizzatori, si sta programmando una seconda edizione con l'Ordine dei Geologi del Lazio, prevista per i primi mesi del 2015. Anche grazie a queste iniziative, il numero dei soci IAH ha superato la soglia dei 200 affiliati, staccando i colleghi francesi e tedeschi e salendo sul podio europeo, poco dietro all'Inghilterra e ancora a distanza dalla Spagna, che supera i 250 soci.
Più che un motivo di innegabile soddisfazione per il Comitato Italiano, vogliamo pensare che a crescere sia l'interesse, la visibilità e la collaborazione in ambito idrogeologico.

Il network internazionale dei soci IAH porta altre occasioni di confronto e di scambio di conoscenze. Il Prof. Craig Simmons del Centro di Ricerca sulle Acque Sotterranee (NCGRT) Australiano sta girando l'Italia presentando nelle sedi di Roma, Napoli, Bologna, Padova, Milano e Torino, le ultime novità in termini di risposta alle sfide imposte dai cambiamenti climatici, dalla salinizzazione delle falde costiere e ad altre problematiche di rinnovato interesse. I dettagli del "tour" sono disponibili sul sito italiano www.iahitaly.it.

Altre occasioni di incontro appena passate dove è stata presente la IAH sono a livello nazionale il convegno di Milano della Società Geologica Italiana, insieme alla Società Italiana di Mineralogia e Petrografia, dove si è svolta una specifica sessione dedicata ai temi idrogeologici, con la partecipazione di circa 30 tra presentazioni orali e poster, arricchita da due keynote-lecture del Prof. Cassiani dell'Università' di Padova e del Prof. Masetti dell'Università di Milano. In ambito internazionale, a Karlovy Vary in Repubblica Ceca si è tenuto l'incontro della Commissione Acque Termali e Minerali della IAH internazionale, con la partecipazione dei membri italiani. La novità di rilievo è che il prossimo anno questo incontro periodico si terrà in Italia, parallelamente al congresso AQUA2015.

Di più ampia partecipazione multidisciplinare è l'incontro ormai periodico di RemTech a Ferrara, dove i temi della bonifica dei siti contaminati vedono a confronto molte diverse professionalità, con l'imprescindibile contributo degli idrogeologi.

Anche ottobre ha le sue occasioni di incontro, a cominciare dal classico appuntamento di GEOFLUID, che caratterizza questo numero di Acque Sotterranee, dove il mondo professionale idrogeologico incontra le aziende, le istituzioni e il mondo della ricerca. Tanti gli appuntamenti a Piacenza, anche in questo caso con la partecipazione di esponenti IAH. Il focus principale è sul workshop "La ricarica delle falde in condizioni controllate", tema che finalmente è oggetto di diverse esperienze qui in Italia e che presumibilmente si diffonderà sempre più, passando dalle applicazioni sperimentali a quelle a pieno campo. Anche in questo caso, il confronto tra le esperienze italiane e quelle europee garantisce un proficuo scambio di idee, soluzioni tecniche, interesse reciproco, con il diretto coinvolgimento delle aziende, delle società tecniche e di consulenza e del mondo della ricerca applicata.

Infine, a testimoniare il ruolo chiave dell'idrogeologia anche nelle politiche dell'Unione Europea, sono in programma a Roma nella prima settimana di ottobre due workshop stret- 
tamente connessi tra loro, in concomitanza con la Presidenza Europea da parte dell'Italia. Tutti i principali attori istituzionali e tecnici della Commissione Europea si sono dati appuntamento presso il Ministero dell'Ambiente, per discutere della direttiva quadro sulle acque e di quella specifica sulle acque sotterranee. Il primo workshop sarà incentrato sul tema delle Drinking Water, trattando sia dei temi sanitari che di quelli di protezione della risorsa. A seguire il secondo workshop, che vede presenti i rappresentanti tecnici di tutti i Paesi membri aderenti al Working Group Groundwater, impegnato a definire la strategia comunitaria di implementazione delle direttive europee, analizzando tra l'altro in dettaglio i delicati temi dei "valori soglia" e dei "Groundwater Dependent Ecosystem", ambienti di importanza ecologica strettamente dipendenti dalle acque sotterranee.

E nuovi appuntamenti "bollono in pentola" per i mesi successivi, a dispetto del "letargo" invernale; un solo cenno alle iniziative che introdurranno l'evento internazionale del 2015, l'EXPO di Milano, dove l'acqua sotterranea può e deve presentarsi da co-protagonista, visto che per sfamare il pianeta, direttamente o indirettamente, l'acqua è e resta indispensabile. Con questa prospettiva, la IAH sta collaborando con Regione Toscana e "Water Right Foundation" per organizzare una giornata di studio sul tema dell'acqua per sfamare il pianeta per la prima settimana di dicembre; anche per questo evento, vi invitiamo a visitare il sito www.iahitaly.it per successivi aggiornamenti.

Da questo breve elenco si evince ancora una volta quanto l'interesse sul tema delle acque sotterranee sia vivo, e soprattutto come sia necessario creare continuamente occasioni di comunicazione, di confronto, di discussione, non tanto per trovarsi tra gli addetti ai lavori, quanto per cercare di svelare il "nostro" lato nascosto del ciclo delle acque, da sempre il più difficile da comunicare.

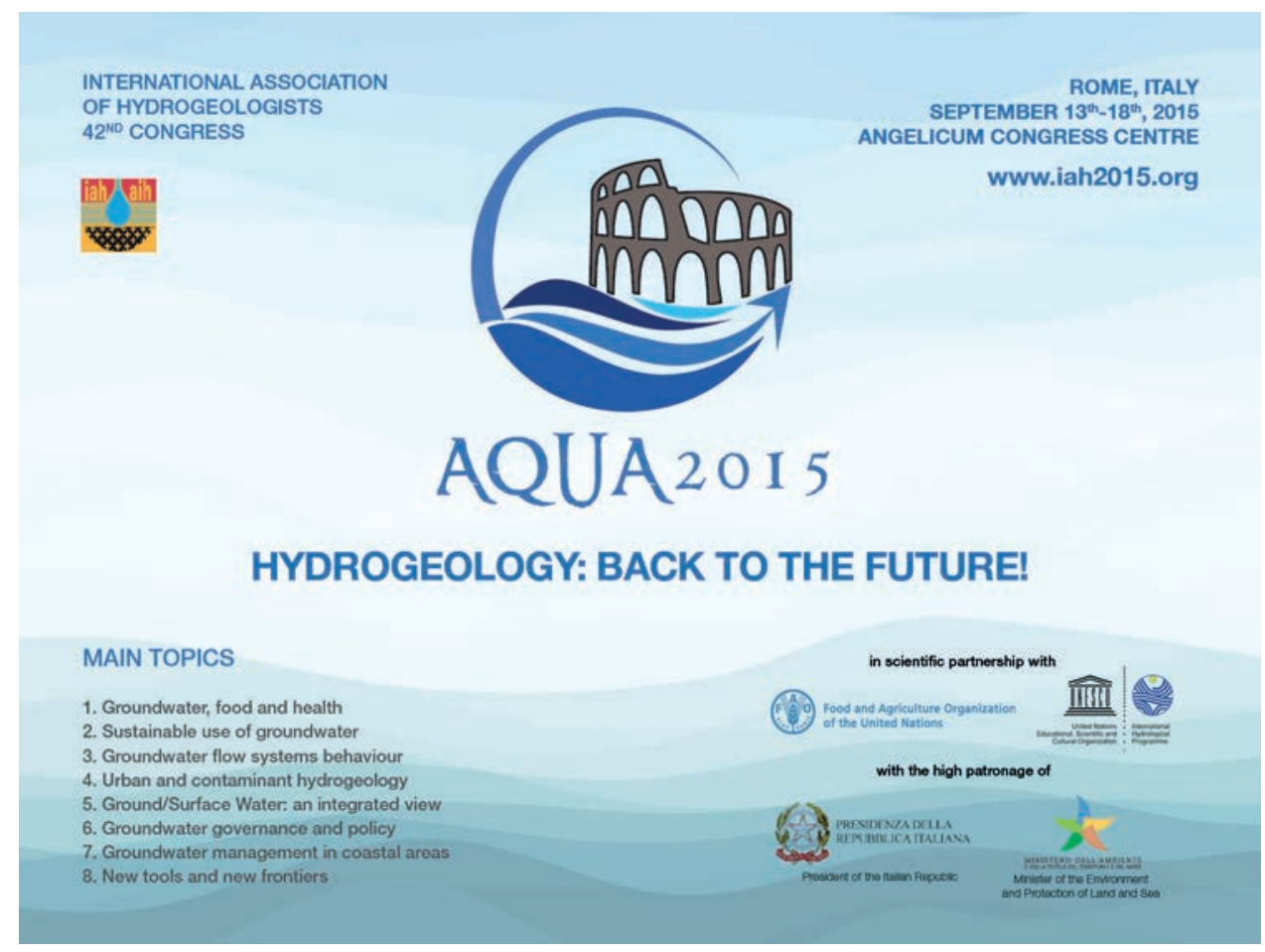

